Supplementary Information

\title{
Palladium-Catalyzed Coupling of Pyrazole Triflates with Arylboronic Acids
}

Curt A. Dvorak, * Dale A. Rudolph, Sandy Ma and Nicholas I. Carruthers

\section{Table of Contents}

General Methods

Characterization Data: 5a-g and 6a-m.

References

${ }^{1} \mathrm{H}$ and ${ }^{13} \mathrm{C}$ NMR 5a

${ }^{1} \mathrm{H}$ and ${ }^{13} \mathrm{C}$ NMR 5b

$\mathrm{S} 10$

${ }^{1} \mathrm{H}$ and ${ }^{13} \mathrm{C}$ NMR 5c

${ }^{1} \mathrm{H}$ and ${ }^{13} \mathrm{C}$ NMR 5d

${ }^{1} \mathrm{H}$ and ${ }^{13} \mathrm{C}$ NMR $5 \mathbf{e}$

${ }^{1} \mathrm{H}$ and ${ }^{13} \mathrm{C}$ NMR $5 \mathbf{f}$

$\mathrm{S} 14$

${ }^{1} \mathrm{H}$ and ${ }^{13} \mathrm{C}$ NMR $5 \mathrm{~g}$

$\mathrm{S} 15$

${ }^{1} \mathrm{H}$ and ${ }^{13} \mathrm{C}$ NMR $\mathbf{6 a}$

${ }^{1} \mathrm{H}$ and ${ }^{13} \mathrm{C}$ NMR $6 \mathbf{b}$

${ }^{1} \mathrm{H}$ and ${ }^{13} \mathrm{C}$ NMR 6e

${ }^{1} \mathrm{H}$ and ${ }^{13} \mathrm{C}$ NMR $6 \mathbf{f}$

${ }^{1} \mathrm{H}$ and ${ }^{13} \mathrm{C}$ NMR 6i 
General Methods. Reagents were purchased from commercial suppliers and used without purification unless noted otherwise. Anhydrous solvents were obtained from a GlassContour Solvent Dispensing System. Anhydrous 1,4-dioxane was purchased from Acros in Acroseal ${ }^{\mathrm{TM}}$ bottles. All reactions were assembled open to air and then placed under nitrogen after the addition of all reagents. Mass spectra were obtained on an Agilent series 1100 MSD using electrospray ionization (ESI) in either positive or negative modes as indicated. Thin-layer chromatography was performed using Merck silica gel $60 \mathrm{~F}_{254} 2.5 \mathrm{~cm} \times 7.5 \mathrm{~cm} 250 \mu \mathrm{m}$ or $5.0 \mathrm{~cm} \times 10.0 \mathrm{~cm} 250 \mu \mathrm{m}$ pre-coated silica gel plates. Preparative thin-layer chromatography was performed using EM Science silica gel $60 \mathrm{~F}_{254} 20 \mathrm{~cm} \times 20 \mathrm{~cm} 0.5 \mathrm{~mm}$ pre-coated plates with a $20 \mathrm{~cm} \times 4 \mathrm{~cm}$ concentrating zone. Chromatography was performed using an ISCO SQ16 instrument with prepacked Redisep ${ }^{\mathrm{TM}}$ silica gel cartridges utilizing gradient elution. ${ }^{1} \mathrm{H}$ and ${ }^{13} \mathrm{C}$ NMR spectra were recorded on Bruker models DPX400, DPX500 or DRX600 spectrometers at 400, 500 and $600 \mathrm{MHz}$ respectively. Chemical shifts are reported in parts per million downfield from an internal standard $\left(\mathrm{Me}_{4} \mathrm{Si}\right)$. IR spectra were collected in a Nicolet Avatar 360 FT-IR spectrometer. Melting points were obtained on either a MelTemp or Electrothermal apparatus and are uncorrected. Analytical reverse phase HPLC was preformed on a Hewlett Packard Series 1100 instrument with an Agilent ZORBAX ${ }^{(3)}$ Bonus RP, column utilizing an acetonitrile/water (0.05\% TFA) gradient.

\section{Characterization Data.}

Trifluoro-methanesulfonic acid 2,5-dimethyl-2H-pyrazol-3-yl ester (5a). Yield:

$1.32 \mathrm{~g}, 62 \% ;{ }^{1} \mathrm{H}$ NMR $\left(500 \mathrm{MHz}, \mathrm{MeOH}_{-} \mathrm{d}_{4}\right) \delta 6.10(\mathrm{~s}, 1 \mathrm{H}), 3.74(\mathrm{~s}, 3 \mathrm{H}), 2.22(\mathrm{~s}, 3 \mathrm{H})$; 
${ }^{13} \mathrm{C}$ NMR $\left(125 \mathrm{MHz}, \mathrm{CDCl}_{3}\right) \delta 148.0,141.6,118.8(\mathrm{q}, J=321.4 \mathrm{~Hz}), 94.7,34.8,14.5$; IR $1433,1247,1211,1131,866,787,636 \mathrm{~cm}^{-1}$; MS (ESI) exact mass calculated for $\mathrm{C}_{6} \mathrm{H}_{7} \mathrm{~F}_{3} \mathrm{~N}_{2} \mathrm{O}_{3} \mathrm{~S}, 244.01$; found, $\mathrm{m} / \mathrm{z} 245.3[\mathrm{M}+\mathrm{H}]^{+}$.

Trifluoro-methanesulfonic acid 5-methyl-2H-pyrazol-3-yl ester (5b). Yield: $0.54 \mathrm{~g}$, $45 \% ;{ }^{1} \mathrm{H}$ NMR $\left(500 \mathrm{MHz}, \mathrm{MeOH}-\mathrm{d}_{4}\right) \delta 6.00(\mathrm{~s}, 1 \mathrm{H}), 2.31(\mathrm{~s}, 3 \mathrm{H}) ;{ }^{13} \mathrm{C}$ NMR $(125 \mathrm{MHz}$, $\left.\mathrm{CDCl}_{3}\right) \delta 153.3,142.3,118.9(\mathrm{q}, J=320.8 \mathrm{~Hz}), 95.2,11.6 ; \mathrm{IR} 1426,1246,1208,1134$, $869 \mathrm{~cm}^{-1}$; MS (ESI) exact mass calculated for $\mathrm{C}_{5} \mathrm{H}_{5} \mathrm{~F}_{3} \mathrm{~N}_{2} \mathrm{O}_{3} \mathrm{~S}, 230.00$; found, $\mathrm{m} / \mathrm{z} 231.3$ $[\mathrm{M}+\mathrm{H}]^{+}$.

Trifluoro-methanesulfonic acid 2-methyl-5-trifluoromethyl-2H-pyrazol-3-yl ester (5c). Yield: $0.62 \mathrm{~g}, 62 \%$; ${ }^{\mathrm{H}} \mathrm{NMR}\left(500 \mathrm{MHz}, \mathrm{MeOH}-\mathrm{d}_{4}\right) \delta 6.72(\mathrm{~s}, 1 \mathrm{H}), 3.92(\mathrm{~s}, 3 \mathrm{H})$; ${ }^{13} \mathrm{C}$ NMR $\left(125 \mathrm{MHz}, \mathrm{CDCl}_{3}\right) \delta 141.7,141.2(\mathrm{q}, J=39.3 \mathrm{~Hz}), 120.1(\mathrm{q}, J=268.7 \mathrm{~Hz})$, $118.5(\mathrm{q}, J=321.5 \mathrm{~Hz}), 94.1,35.8$; IR 1216, 1128, 1097, 856, $602 \mathrm{~cm}^{-1}$; MS (ESI) exact mass calculated for $\mathrm{C}_{6} \mathrm{H}_{4} \mathrm{~F}_{6} \mathrm{~N}_{2} \mathrm{O}_{3} \mathrm{~S}, 297.98$; found, m/z $299.3[\mathrm{M}+\mathrm{H}]^{+}$.

\section{Trifluoro-methanesulfonic acid 5-methyl-2-phenyl-2H-pyrazol-3-yl ester (5d). ${ }^{1}$}

Yield: $1.83 \mathrm{~g}, 92 \% ;{ }^{1} \mathrm{H}$ NMR $\left(500 \mathrm{MHz}, \mathrm{MeOH}-\mathrm{d}_{4}\right) \delta 7.58-7.48(\mathrm{~m}, 5 \mathrm{H}), 6.35(\mathrm{~s}, 1 \mathrm{H})$, $2.33(\mathrm{~s}, 3 \mathrm{H}) ;{ }^{13} \mathrm{C}$ NMR $(125 \mathrm{MHz}, \mathrm{CDCl} 3) \delta 149.3,140.9,136.9,129.4,128.4,123.7$, $118.5(\mathrm{q}, J=321.5 \mathrm{~Hz}), 96.5,14.5 ;$ IR $1434,1212,1130,853,758,665 \mathrm{~cm}^{-1} ;$ MS (ESI) exact mass calculated for $\mathrm{C}_{11} \mathrm{H}_{9} \mathrm{~F}_{3} \mathrm{~N}_{2} \mathrm{O}_{3} \mathrm{~S}, 306.03$; found, $\mathrm{m} / \mathrm{z} 307.3[\mathrm{M}+\mathrm{H}]^{+}$. Trifluoro-methanesulfonic acid 2-phenyl-5-trifluoromethyl-2H-pyrazol-3-yl ester (5e). Yield: $0.60 \mathrm{~g}, 60 \% ;{ }^{1} \mathrm{H}$ NMR $\left(500 \mathrm{MHz}, \mathrm{MeOH}-\mathrm{d}_{4}\right) \delta 7.63-7.57(\mathrm{~m}, 5 \mathrm{H}), 6.98(\mathrm{~s}$, $1 \mathrm{H}) ;{ }^{13} \mathrm{C}$ NMR $\left(125 \mathrm{MHz}, \mathrm{CDCl}_{3}\right) \delta 142.4(\mathrm{q}, J=40.3 \mathrm{~Hz}), 141.3,135.8,129.8$, $129.6,124.2,120.4(\mathrm{q}, J=269.4 \mathrm{~Hz}), 118.6(\mathrm{q}, J=321.7 \mathrm{~Hz}), 95.4 ; \mathrm{IR} 1214,1128,845$, 
$764,647 \mathrm{~cm}^{-1}$; MS (ESI) exact mass calculated for $\mathrm{C}_{11} \mathrm{H}_{6} \mathrm{~F}_{6} \mathrm{~N}_{2} \mathrm{O}_{3} \mathrm{~S}, 360.00$; found, $\mathrm{m} / \mathrm{z}$ $361.3[\mathrm{M}+\mathrm{H}]^{+}$.

Trifluoro-methanesulfonic acid 2-methyl-4,5,6,7-tetrahydro-2H-indazol-3-yl ester (5f). Yield: $0.88 \mathrm{~g}, 88 \%$; ${ }^{1} \mathrm{H}$ NMR $\left(400 \mathrm{MHz}, \mathrm{MeOH}-\mathrm{d}_{4}\right) \delta 3.71(\mathrm{~s}, 3 \mathrm{H}), 2.57(\mathrm{t}, J=$ $6.1 \mathrm{~Hz}, 2 \mathrm{H}), 2.47(\mathrm{t}, J=6.1 \mathrm{~Hz}, 2 \mathrm{H}), 1.83-1.70(\mathrm{~m}, 4 \mathrm{H}) ;{ }^{13} \mathrm{C} \mathrm{NMR}\left(125 \mathrm{MHz} \mathrm{CDCl}_{3}\right) \delta$ $148.7,137.0,118.4(\mathrm{q}, J=320.5 \mathrm{~Hz}), 105.2,34.8,23.6,22.7,22.5,19.1$; IR 1211, 1134, 887, 843, 814, $\mathrm{cm}^{-1}$; MS (ESI) exact mass calculated for $\mathrm{C}_{9} \mathrm{H}_{11} \mathrm{~F}_{3} \mathrm{~N}_{2} \mathrm{O}_{3} \mathrm{~S}, 284.04$; found, $\mathrm{m} / \mathrm{z} 285.3[\mathrm{M}+\mathrm{H}]^{+}$.

Trifluoro-methanesulfonic acid 2-phenyl-4,5,6,7-tetrahydro-2H-indazol-3-yl ester

(5g). Yield: $0.94 \mathrm{~g}, 94 \% ;{ }^{1} \mathrm{H}$ NMR $\left(500 \mathrm{MHz}, \mathrm{MeOH}-\mathrm{d}_{4}\right) \delta 7.57-7.52(\mathrm{~m}, 2 \mathrm{H}), 7.51-7.45$ $(\mathrm{m}, 3 \mathrm{H}), 2.71(\mathrm{t}, J=6.3 \mathrm{~Hz}, 2 \mathrm{H}), 2.61(\mathrm{t}, J=6.3 \mathrm{~Hz}, 2 \mathrm{H}), 1.92-1.86(\mathrm{~m}, 2 \mathrm{H}), 1.85-1.80(\mathrm{~m}$, $2 \mathrm{H}) ;{ }^{13} \mathrm{C} \mathrm{NMR}\left(125 \mathrm{MHz}, \mathrm{CDCl}_{3}\right) \delta 150.2,137.1,136.6,129.2,127.9,123.6,118.1(\mathrm{q}$, $J=321.7 \mathrm{~Hz}), 107.2,23.8,22.7,22.5,19.4$; IR 1428, 1238, 1211, 1134, 867, 839, 758, $603 \mathrm{~cm}^{-1}$; MS (ESI) exact mass calculated for $\mathrm{C}_{14} \mathrm{H}_{13} \mathrm{~F}_{3} \mathrm{~N}_{2} \mathrm{O}_{3} \mathrm{~S}, 346.06$; found, $\mathrm{m} / \mathrm{z} 347.3$ $[\mathrm{M}+\mathrm{H}]^{+}$

1,3-Dimethyl-5-phenyl-1 H-pyrazole (6a). ${ }^{2}$ Yield: $0.143 \mathrm{~g}, 83 \% ;{ }^{1} \mathrm{H}$ NMR $(500 \mathrm{MHz}$, $\left.\mathrm{MeOH}-\mathrm{d}_{4}\right) \delta 7.50-7.41(\mathrm{~m}, 5 \mathrm{H}), 6.14(\mathrm{~s}, 1 \mathrm{H}), 3.78(\mathrm{~s}, 3 \mathrm{H}), 2.25(\mathrm{~s}, 3 \mathrm{H}) ;{ }^{13} \mathrm{C}$ NMR $(125$ $\left.\mathrm{MHz}, \mathrm{CDCl}_{3}\right) \delta 147.5,144.3,130.9,128.6,128.5,128.2,105.5,37.0,13.4 ;$ IR 1469, 775, $755,698 \mathrm{~cm}^{-1}$; MS (ESI) exact mass calculated for $\mathrm{C}_{11} \mathrm{H}_{12} \mathrm{~N}_{2}, 172.10$; found, $\mathrm{m} / \mathrm{z} 173.4$ $[\mathrm{M}+\mathrm{H}]^{+}$

3-Methyl-5-phenyl-1H-pyrazole (6b). ${ }^{3}$ Yield: $0.127 \mathrm{~g}, 81 \% ;{ }^{1} \mathrm{H}$ NMR $(500 \mathrm{MHz}$, MeOH-d 4$) \delta 7.71(\mathrm{bs}, 2 \mathrm{H}), 7.38(\mathrm{t}, J=7.4 \mathrm{~Hz}, 2 \mathrm{H}), 7.32-7.27(\mathrm{~m}, 1 \mathrm{H}), 6.40(\mathrm{~s}, 1 \mathrm{H}), 2.32$ $(\mathrm{s}, 3 \mathrm{H}) ;{ }^{13} \mathrm{C}$ NMR $\left(125 \mathrm{MHz}, \mathrm{CDCl}_{3}\right) \delta 133.8,131.4,128.8,128.3,127.6,125.8,102.6$, 
11.8; IR 1334, 1306, 762, $689 \mathrm{~cm}^{-1}$; MS (ESI) exact mass calculated for $\mathrm{C}_{10} \mathrm{H}_{10} \mathrm{~N}_{2}$, 158.08; found, $\mathrm{m} / \mathrm{z} 159.4[\mathrm{M}+\mathrm{H}]^{+}$.

1-Methyl-5-phenyl-3-trifluoromethyl-1H-pyrazole (6c). Yield: $0.179 \mathrm{~g}, 79 \% ;{ }^{1} \mathrm{H}$ NMR $\left(400 \mathrm{MHz}, \mathrm{CDCl}_{3}\right) \delta 7.52-7.46(\mathrm{~m}, 3 \mathrm{H}), 7.43-7.40(\mathrm{~m}, 2 \mathrm{H}), 6.56(\mathrm{~s}, 1 \mathrm{H}), 3.92(\mathrm{~s}$, $3 \mathrm{H}) ;{ }^{13} \mathrm{C}$ NMR $\left(125 \mathrm{MHz}, \mathrm{CDCl}_{3}\right) \delta 145.3,141.7(\mathrm{q}, J=37.9 \mathrm{~Hz}), 129.5,129.5,129.1$, $129.0,121.6(\mathrm{q}, J=268.5 \mathrm{~Hz}), 104.5,38.2 ;$ IR $1173,1142,1111,974,701 \mathrm{~cm}^{-1}$; MS (ESI) exact mass calculated for $\mathrm{C}_{11} \mathrm{H}_{9} \mathrm{~F}_{3} \mathrm{~N}_{2}, 226.07$; found, $\mathrm{m} / \mathrm{z} 227.4[\mathrm{M}+\mathrm{H}]^{+}$.

3-Methyl-1,5-diphenyl-1H-pyrazole (6d). ${ }^{\mathbf{4 5}}$ Yield: $0.189 \mathrm{~g}, 81 \%$; ${ }^{\mathrm{H}} \mathrm{NMR}(500 \mathrm{MHz}$, MeOH-d $\left.{ }_{4}\right) \delta 7.40-7.34(\mathrm{~m}, 3 \mathrm{H}), 7.31-7.27(\mathrm{~m}, 3 \mathrm{H}), 7.26-7.19(\mathrm{~m}, 4 \mathrm{H}), 6.41(\mathrm{~s}, 1 \mathrm{H}) ; 2.35$ $(\mathrm{s}, 3 \mathrm{H}) ;{ }^{13} \mathrm{C} \mathrm{NMR}\left(125 \mathrm{MHz}, \mathrm{CDCl}_{3}\right) \delta 149.6,143.9,140.4,130.9,129.0,128.8,128.6$, 128.2, 127.3, 125.3, 107.9, 13.8; IR 1504, 759, $693 \mathrm{~cm}^{-1}$; MS (ESI) exact mass calculated for $\mathrm{C}_{16} \mathrm{H}_{14} \mathrm{~N}_{2}, 234.12$; found, $\mathrm{m} / \mathrm{z} 235.4[\mathrm{M}+\mathrm{H}]^{+}$.

1,5-Diphenyl-3-trifluoromethyl-1H-pyrazole (6e). ${ }^{6}$ Yield: $0.212 \mathrm{~g}, 74 \%$; ${ }^{1} \mathrm{H}$ NMR $\left(500 \mathrm{MHz}, \mathrm{MeOH}-\mathrm{d}_{4}\right) \delta$ 7.46-7.42 (m, 3H), 7.39-7.31 (m, 5H), 7.29-7.26 (m, 2H), 6.93 $(\mathrm{s}, 1 \mathrm{H}) ;{ }^{13} \mathrm{C} \mathrm{NMR}\left(125 \mathrm{MHz}, \mathrm{CDCl}_{3}\right) \delta 144.9,143.6(\mathrm{t}, J=38.5 \mathrm{~Hz}), 139.5,129.4,129.3$, 129.2, 129.0, 128.9, 128.6, 125.7, 121.5 (q, $J=269.4 \mathrm{~Hz}), 105.8$; IR 1234, 1168, 1120 , $1099,977,762,694 \mathrm{~cm}^{-1}$; MS (ESI) exact mass calculated for $\mathrm{C}_{16} \mathrm{H}_{11} \mathrm{~F}_{3} \mathrm{~N}_{2}, 288.09$; found, $\mathrm{m} / \mathrm{z} 289.4[\mathrm{M}+\mathrm{H}]^{+}$.

2-Methyl-3-phenyl-4,5,6,7-tetrahydro-2H-indazole (6f). Yield: $0.150 \mathrm{~g}, 71 \% ;{ }^{1} \mathrm{H}$ NMR $\left(500 \mathrm{MHz}, \mathrm{MeOH}-\mathrm{d}_{4}\right) \delta 7.50(\mathrm{t}, J=8.2 \mathrm{~Hz}, 2 \mathrm{H}), 7.43(\mathrm{t}, J=7.4 \mathrm{~Hz}, 2 \mathrm{H}), 7.38(\mathrm{~d}, J$ $=8.2 \mathrm{~Hz}, 1 \mathrm{H},), 3.73(\mathrm{~s}, 3 \mathrm{H}), 2.65(\mathrm{t}, J=6.3 \mathrm{~Hz}, 2 \mathrm{H}),, 2.47(\mathrm{t}, J=6.3 \mathrm{~Hz}, 2 \mathrm{H}), 1.88-1.83$ $(\mathrm{m}, 2 \mathrm{H}), 1.78-1.72(\mathrm{~m}, 2 \mathrm{H}) ;{ }^{13} \mathrm{C} \mathrm{NMR}\left(\mathrm{CDCl}_{3}, 100 \mathrm{MHz}\right) \delta 148.4,139.6,130.9,129.3$, 
$128.8,128.2,114.9,37.2,23.8,23.6,23.6,21.4 ;$ IR 1489, 1372, 766, $702 \mathrm{~cm}^{-1}$; MS (ESI) exact mass calculated for $\mathrm{C}_{14} \mathrm{H}_{16} \mathrm{~N}_{2}, 212.13$; found, $\mathrm{m} / \mathrm{z} 213.4[\mathrm{M}+\mathrm{H}]^{+}$.

2,3-Diphenyl-4,5,6,7-tetrahydro-2H-indazole (6g). ${ }^{7}$ Yield: 0.202g, 74\%; ${ }^{1} \mathrm{H}$ NMR $\left(500 \mathrm{MHz}, \mathrm{MeOH}-\mathrm{d}_{4}\right) \delta$ 7.35-7.28 (m, 5H), 7.22-7.14 (m, 4H), 6.81-6.74 (m, 1H), $2.76(\mathrm{t}$, $J=6.3 \mathrm{~Hz}, 2 \mathrm{H}), 2.60(\mathrm{t}, J=6.3 \mathrm{~Hz}, 2 \mathrm{H}), 1.95-1.90(\mathrm{~m}, 2 \mathrm{H}), 1.84-1.80(\mathrm{~m}, 2 \mathrm{H}) ;{ }^{13} \mathrm{C}$ NMR $\left(125 \mathrm{MHz}, \mathrm{CDCl}_{3}\right) \delta 150.5,140.6,138.6,131.0,129.4,128.9,128.5,127.9,126.7,124.9$, 117.0, 23.7, 23.7, 23.5, 21.7; IR 1592, 1503, 1363, 762, 754, $692 \mathrm{~cm}^{-1}$; MS (ESI) exact mass calculated for $\mathrm{C}_{19} \mathrm{H}_{18} \mathrm{~N}_{2}, 274.15$; found, m/z $275.4[\mathrm{M}+\mathrm{H}]^{+}$.

5-(4-Fluoro-phenyl)-1,3-dimethyl-1H-pyrazole (6h). Yield: $0.1085 \mathrm{~g}, 85 \%$; ${ }^{\mathrm{H}} \mathrm{H}$ NMR $\left(600 \mathrm{MHz}, \mathrm{CDCl}_{3}\right) \delta$ 7.37-7.33 (m, 2H), 7.15-7.10 (m, 2H), $6.05(\mathrm{~s}, 1 \mathrm{H}), 3.78(\mathrm{~s}, 3 \mathrm{H})$, $2.28(\mathrm{~s}, 3 \mathrm{H}) ;{ }^{13} \mathrm{C} \mathrm{NMR}\left(150 \mathrm{MHz}, \mathrm{CDCl}_{3}\right) \delta 162.6(\mathrm{~d}, \mathrm{~J}=248.9 \mathrm{~Hz}), 147.6,144.3,130.4$, $(\mathrm{d}, \mathrm{J}=8.0 \mathrm{~Hz}), 126.9(\mathrm{~d}, \mathrm{~J}=3.4 \mathrm{~Hz}), 115.5,(\mathrm{~d}, J=21 \mathrm{~Hz}), 105.6,36.9,13.4$; IR 1503 , $1219,1157,1002,838,821,636 \mathrm{~cm}^{-1}$; MS (ESI) exact mass calculated for $\mathrm{C}_{11} \mathrm{H}_{11} \mathrm{FN}_{2}$, 190.22; found, $\mathrm{m} / \mathrm{z} 191.4[\mathrm{M}+\mathrm{H}]^{+}$.

5-(4-Methoxy-phenyl)-1,3-dimethyl-1H-pyrazole (6i). ${ }^{8}$ Yield: $0.181 \mathrm{~g}, 90 \%$; ${ }^{1} \mathrm{H}$ NMR $\left(500 \mathrm{MHz}, \mathrm{CDCl}_{3}\right) \delta 7.33(\mathrm{~d}, J=8.8 \mathrm{~Hz}, 2 \mathrm{H}), 6.98(\mathrm{~d}, J=8.8 \mathrm{~Hz}, 2 \mathrm{H}), 6.04(\mathrm{~s}, 1 \mathrm{H}), 3.86$ (s, 3H), 3.78 (s, 3H), 2.30 (s, 3H); ${ }^{13} \mathrm{C}$ NMR (125 MHz, $\left.\mathrm{CDCl}_{3}\right) \delta 159.8,147.7$, 144.4, 130.1, 123.6, 114.2, 105.4, 55.5, 37.1, 13.6; IR 1504, 1245, 1172, 1035, 835, 822, 795 $\mathrm{cm}^{-1}$; MS (ESI) exact mass calculated for $\mathrm{C}_{12} \mathrm{H}_{14} \mathrm{~N}_{2} \mathrm{O}, 202.11$; found, m/z $203.4[\mathrm{M}+\mathrm{H}]^{+}$. 1,3-Dimethyl-5-o-tolyl-1H-pyrazole (6j). Yield: $0.147 \mathrm{~g}, 79 \%$; ${ }^{1} \mathrm{H}$ NMR $(500 \mathrm{MHz}$, $\left.\mathrm{CDCl}_{3}\right) \delta 7.33-7.26(\mathrm{~m}, 2 \mathrm{H}), 7.24-7.21(\mathrm{~m}, 1 \mathrm{H}), 7.19-7.16(\mathrm{~m}, 1 \mathrm{H}), 5.96(\mathrm{~s}, 1 \mathrm{H}), 3.57(\mathrm{~s}$, $3 \mathrm{H}), 2.30(\mathrm{~s}, 3 \mathrm{H}), 2.17(\mathrm{~s}, 3 \mathrm{H}) ;{ }^{13} \mathrm{C} \mathrm{NMR}\left(125 \mathrm{MHz}, \mathrm{CDCl}_{3}\right) \delta 147.6,143.4,137.6$, 
$130.9,130.3,129.0,125.8,106.1,36.3,19.9,13.6 ;$ IR 1493, 770, 750, $725 \mathrm{~cm}^{-1}$; MS

(ESI) exact mass calculated for $\mathrm{C}_{12} \mathrm{H}_{14} \mathrm{~N}_{2}$ 186.12, found, $\mathrm{m} / \mathrm{z} 187.4[\mathrm{M}+\mathrm{H}]^{+}$.

1-[4-(2,5-Dimethyl-2H-pyrazol-3-yl)-phenyl]-ethanone (6k). Yield: $0.15 \lg , 71 \% ;{ }^{1} \mathrm{H}$ $\operatorname{NMR}\left(500 \mathrm{MHz}, \mathrm{CDCl}_{3}\right) \delta 8.04(\mathrm{~d}, J=8.5 \mathrm{~Hz}, 2 \mathrm{H}), 7.52(\mathrm{~d}, J=8.5 \mathrm{~Hz}, 2 \mathrm{H}), 6.17(\mathrm{~s}$, $1 \mathrm{H}), 3.86(\mathrm{~s}, 3 \mathrm{H}), 2.65(\mathrm{~s}, 3 \mathrm{H}), 2.31(\mathrm{~s}, 3 \mathrm{H}) ;{ }^{13} \mathrm{C} \mathrm{NMR}\left(125 \mathrm{MHz}, \mathrm{CDCl}_{3}\right) \delta$ 197.6, 148.1, $143.4,136.8,135.6,128.9,128.8,106.4,37.6,26.8,13.6$; IR 1687, 1607, 1355, 1264, $1001,839,816 \mathrm{~cm}^{-1}$; MS (ESI) exact mass calculated for $\mathrm{C}_{13} \mathrm{H}_{14} \mathrm{~N}_{2} \mathrm{O}, 214.11$; found, $\mathrm{m} / \mathrm{z}$ $215.4[\mathrm{M}+\mathrm{H}]^{+}$.

5-(4-Methoxy-phenyl)-3-methyl-1-phenyl-1H-pyrazole (61). ${ }^{2}$ Yield: $0.101 \mathrm{~g}, 76 \% ;{ }^{1} \mathrm{H}$ $\operatorname{NMR}\left(500 \mathrm{MHz}, \mathrm{CDCl}_{3}\right) \delta 7.34-7.25(\mathrm{~m}, 5 \mathrm{H}), 7.14(\mathrm{~d}, J=9.0 \mathrm{~Hz}, 2 \mathrm{H}), 6.81(\mathrm{~d}, J=9.0$ $\mathrm{Hz}, 2 \mathrm{H}), 6.25(\mathrm{~s}, 1 \mathrm{H}), 3.80(\mathrm{~s}, 3 \mathrm{H}), 2.38(\mathrm{~s}, 3 \mathrm{H}) ;{ }^{13} \mathrm{C} \mathrm{NMR}\left(125 \mathrm{MHz}, \mathrm{CDCl}_{3}\right) \delta 159.6$, 149.6, 143.8, 140.5, 130.1, 129.0, 127.2, 125.4, 114.1, 107.4, 55.4, 13.8; IR 1509, 1499, $1247,1177,1032,834,792,762,694 \mathrm{~cm}^{-1}$; MS (ESI) exact mass calculated for $\mathrm{C}_{17} \mathrm{H}_{16} \mathrm{~N}_{2} \mathrm{O}$ 264.13, found, $\mathrm{m} / \mathrm{z} 265.5[\mathrm{M}+\mathrm{H}]^{+}$.

3-Methyl-1-phenyl-5-o-tolyl-1H-pyrazole (6m). Yield: 0.229g, 92\%; ${ }^{1} \mathrm{H}$ NMR (500 $\left.\mathrm{MHz}, \mathrm{CDCl}_{3}\right) \delta$ 7.29-7.16(m, 9H), $6.21(\mathrm{~s}, 1 \mathrm{H}), 2.42(\mathrm{~s}, 3 \mathrm{H}), 2.01(\mathrm{~s}, 3 \mathrm{H}) ;{ }^{13} \mathrm{C}$ NMR $\left(125 \mathrm{MHz}, \mathrm{CDCl}_{3}\right) \delta 149.4,143.1,140.5,137.2,131.3,130.7,130.5,128.9,128.9,126.6$, $125.9,123.7,108.9,20.1,13.9 ;$ IR 1503, 1493, 1361, 782, 766, 755, $687 \mathrm{~cm}^{-1}$; MS (ESI) exact mass calculated for $\mathrm{C}_{17} \mathrm{H}_{16} \mathrm{~N}_{2}, 248.13$, found, $\mathrm{m} / \mathrm{z} 249.4[\mathrm{M}+\mathrm{H}]^{+}$.

\section{References.}

' Ying, W.; DesMarteau, D.D.; Xu, Z. and Witz, M. J. Fluorine Chem. 2000, 102, 135

${ }^{2}$ Alberola, A.; Bleye, L.; Gonzalez-Ortega, A.; Sadaba, M.; Sanudo, M. Heterocycles 2001, 55(2), 331-351. 
${ }^{3}$ Alberola, A.; Calvo,L.; Ortega, A.; Sadaba, M.; Sanudo, M.; Granda, S.; Rodriguez, E. Heterocycles 1999, 5l(11), 2675-2686.

${ }^{4}$ Texier-Boullet, F.; Klein, B.; Hamelin, J. Synthesis 1986, (5), 409-11. 5

${ }^{6}$ Sloop, J.; Bumgardner, C.; Loehle, W. J. of Fluorine Chem. 2002, 118(1-2), 135-147.

${ }^{7}$ Lee, Ka Young; Kim, Jeong Mi; Kim, Jae Nyoung. Tetrahedron Letters 2003, 44(35), $6737-6740$.

${ }^{8}$ Valduga, Claudete J.; Braibante, Hugo S.; Braibante, Mara E. F. J. Heterocyclic Chem. 1998, 35(1), 189-192. 
1.0

$\left.\sum^{N}\right\rangle^{H}-07 \theta$

after chromas.

,

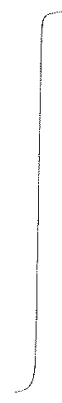
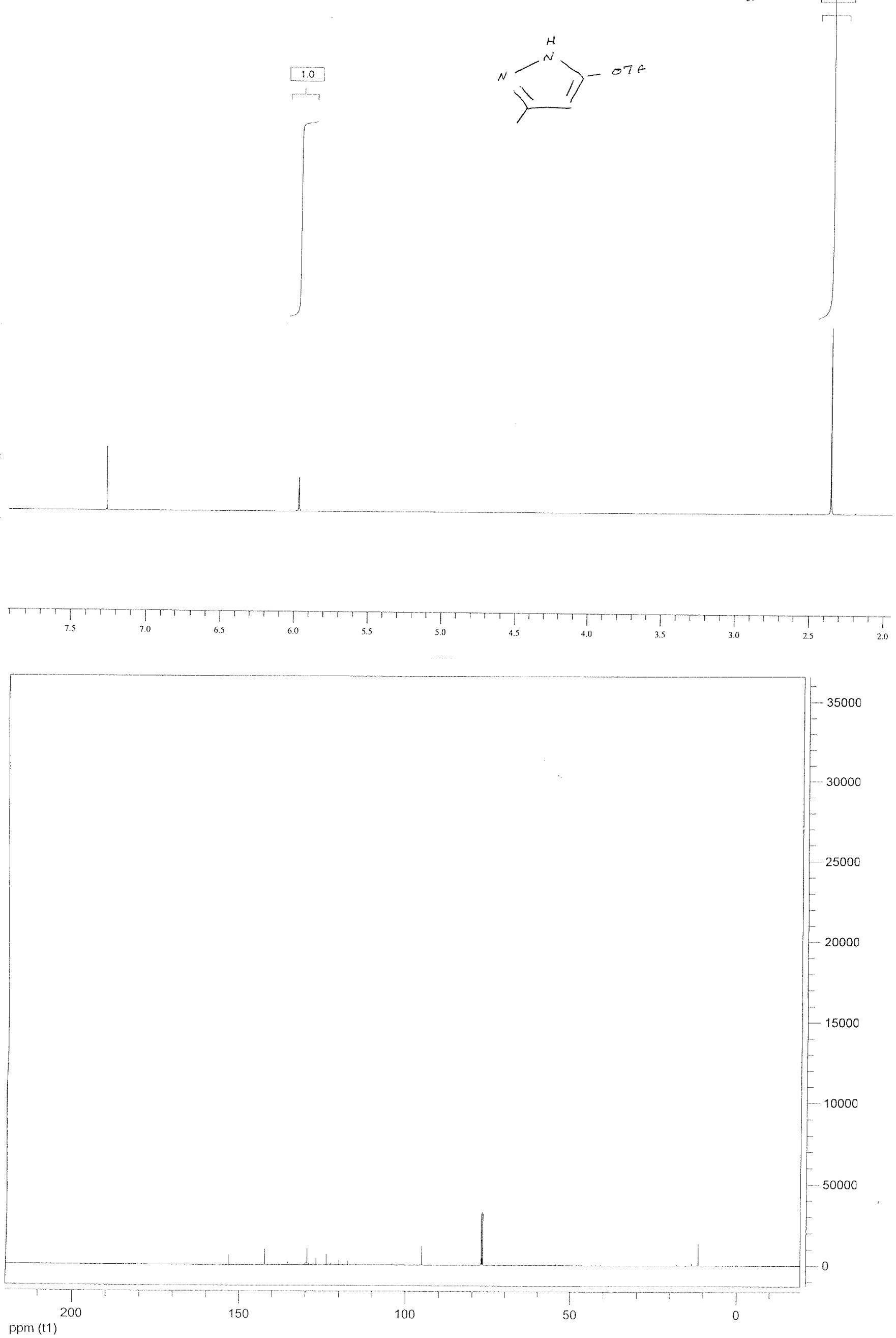

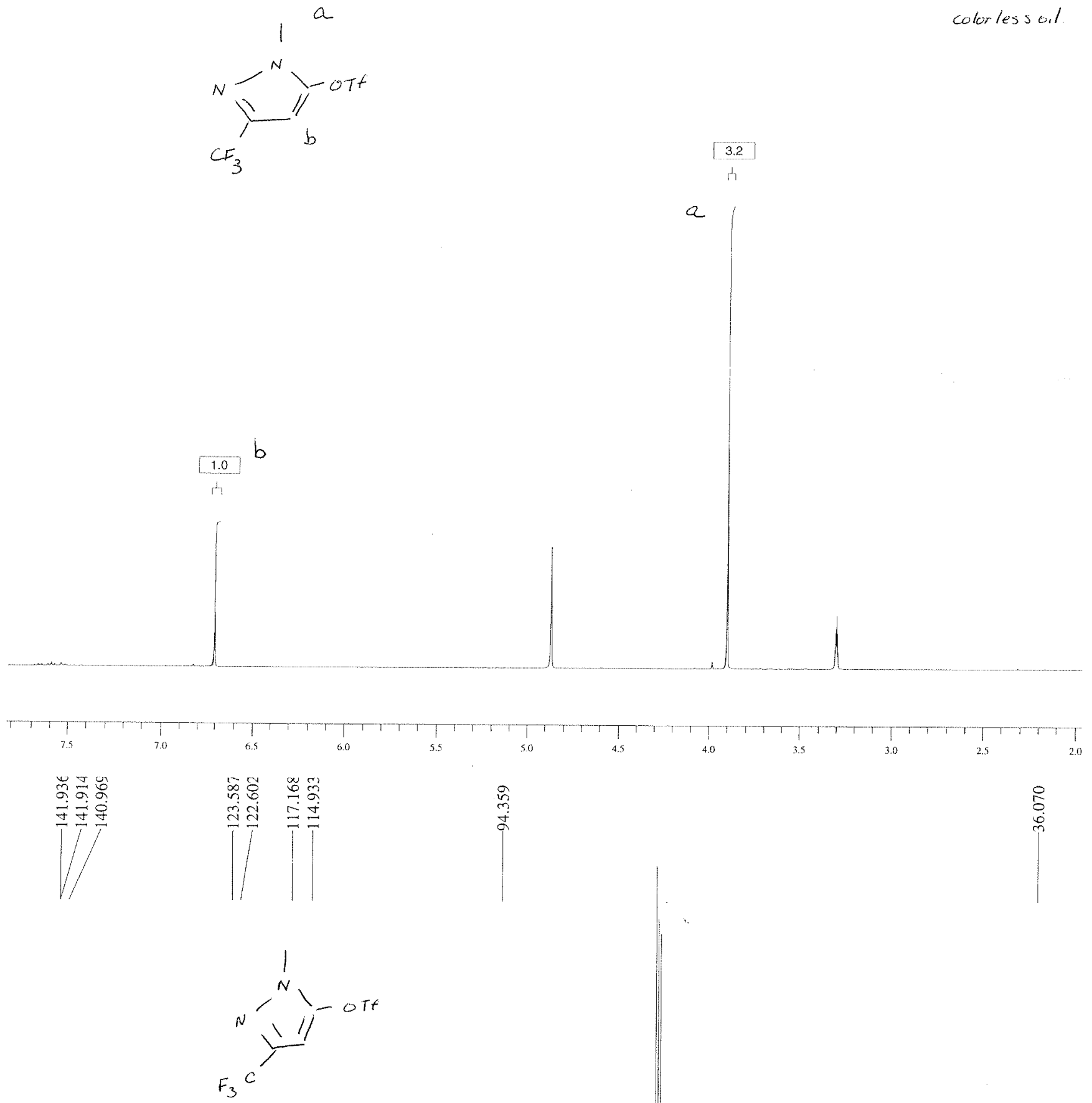



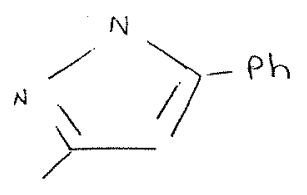

4.7
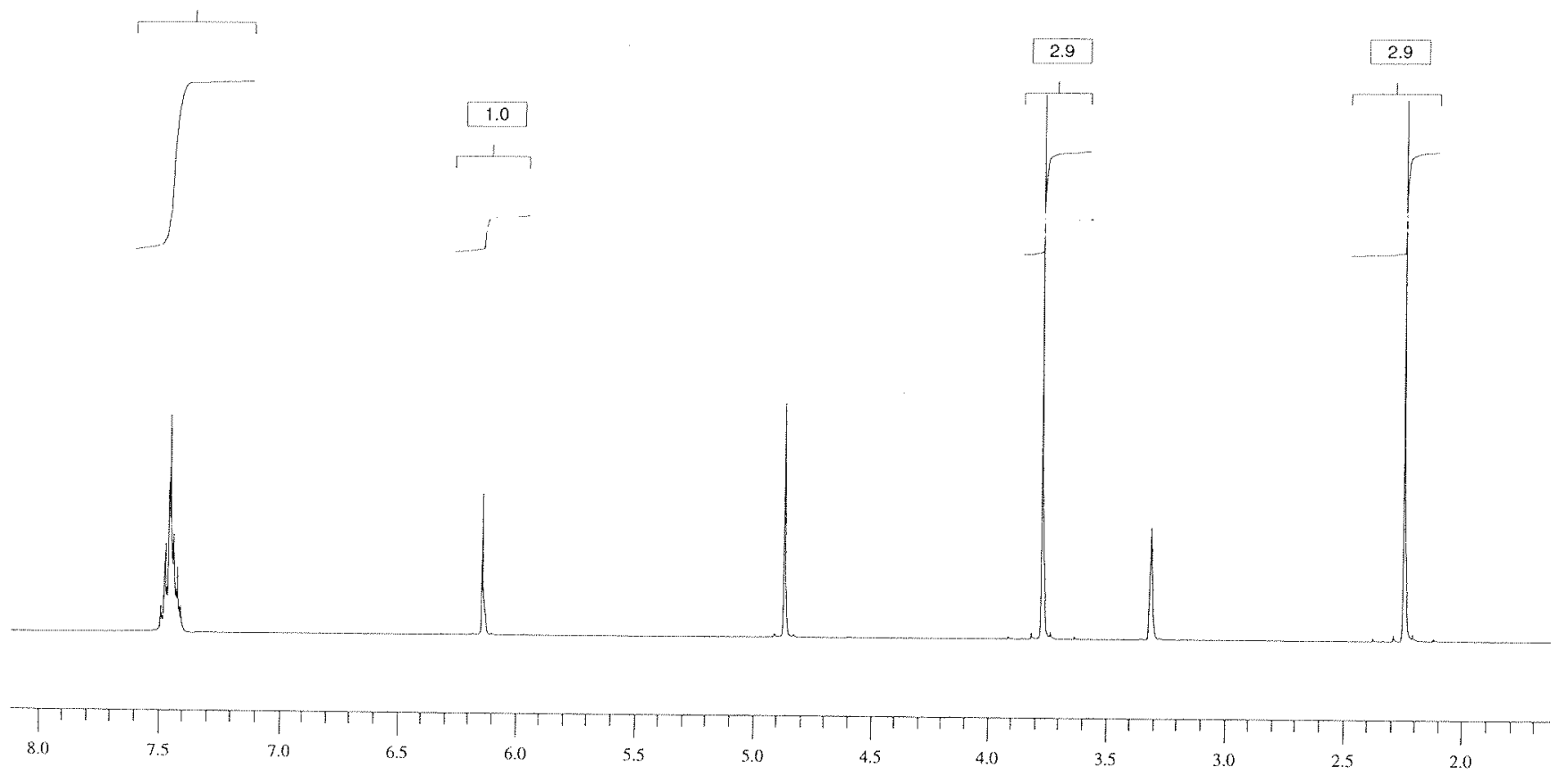

$570 \cdots 696$

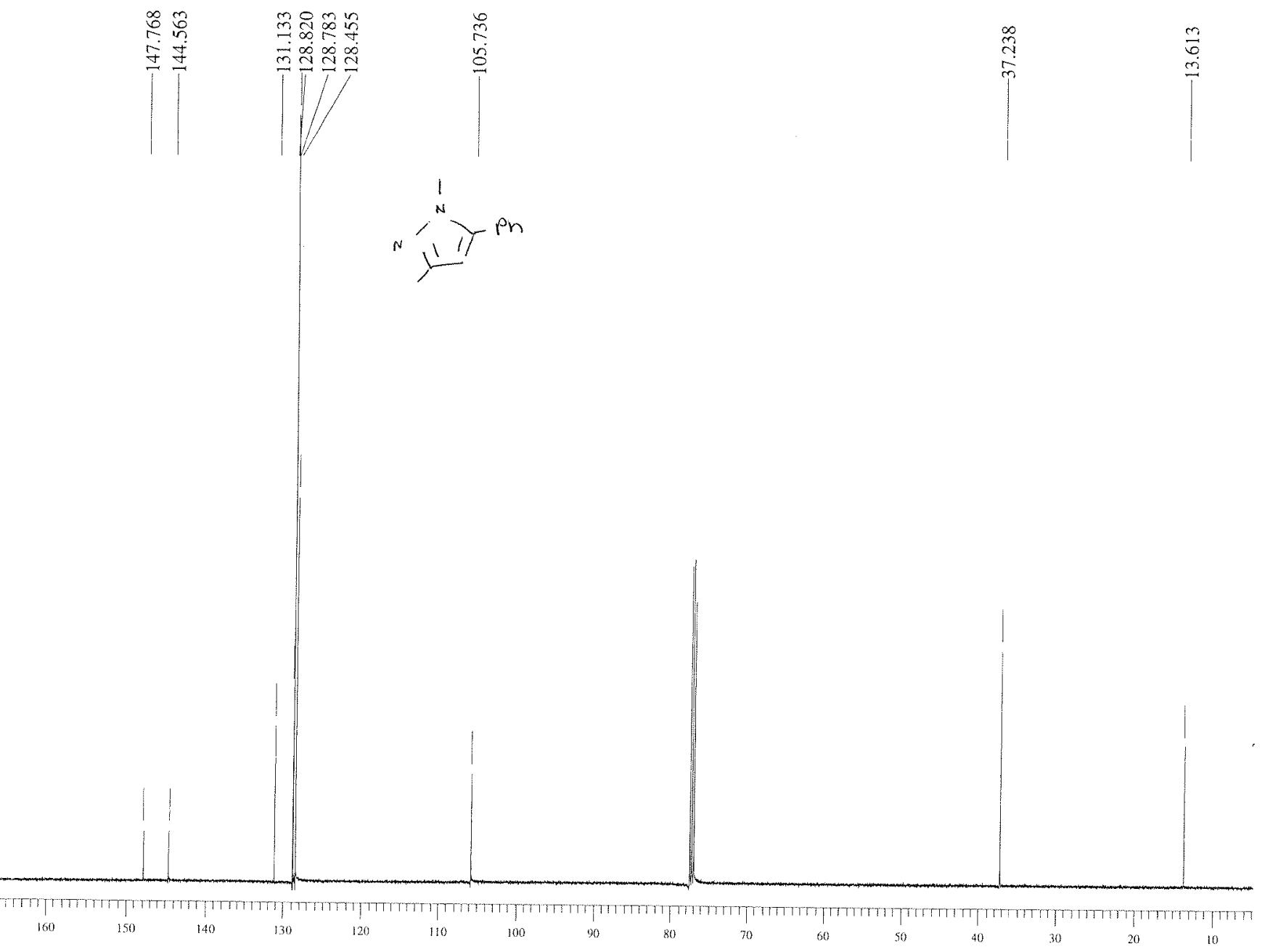




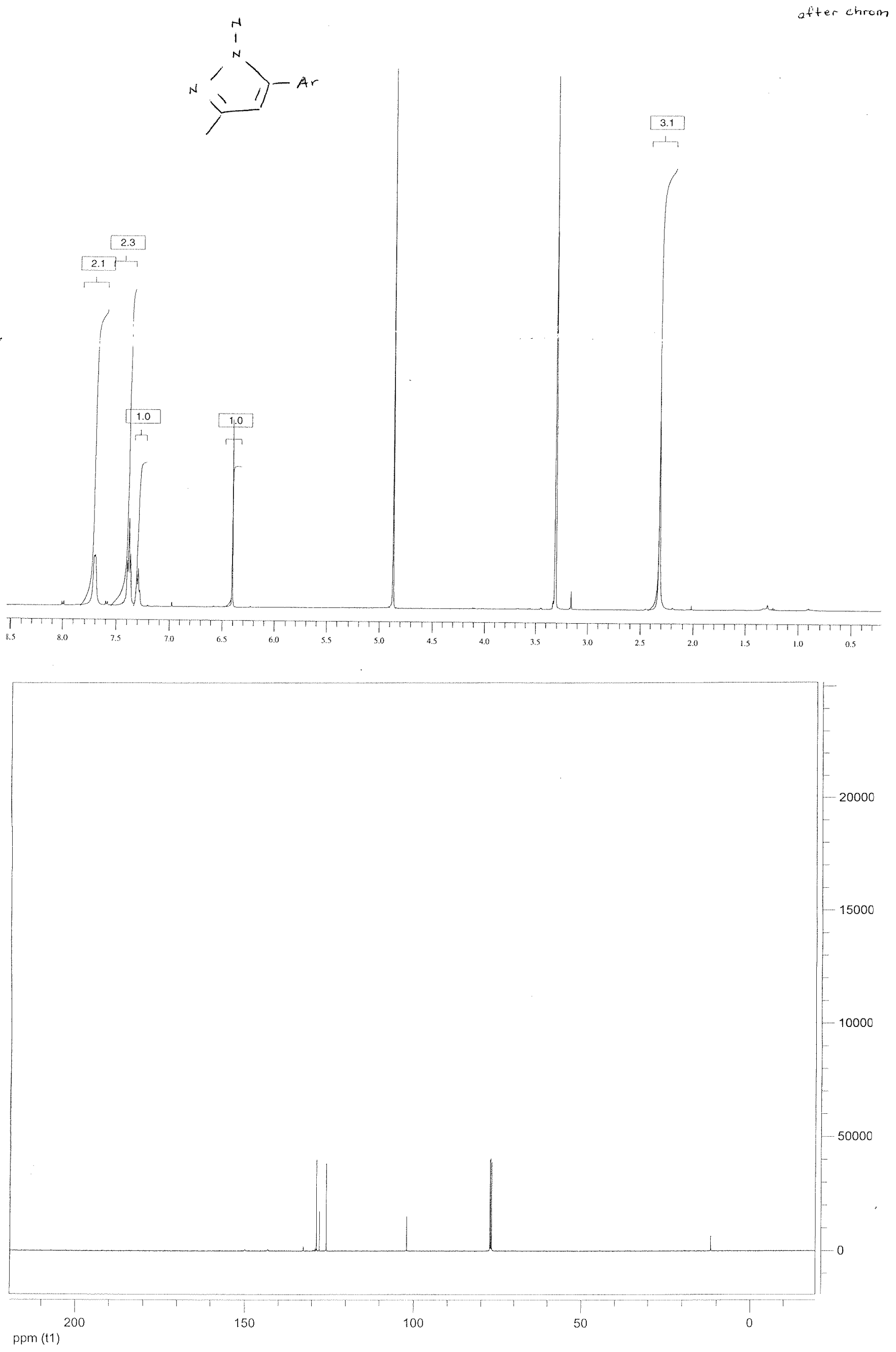



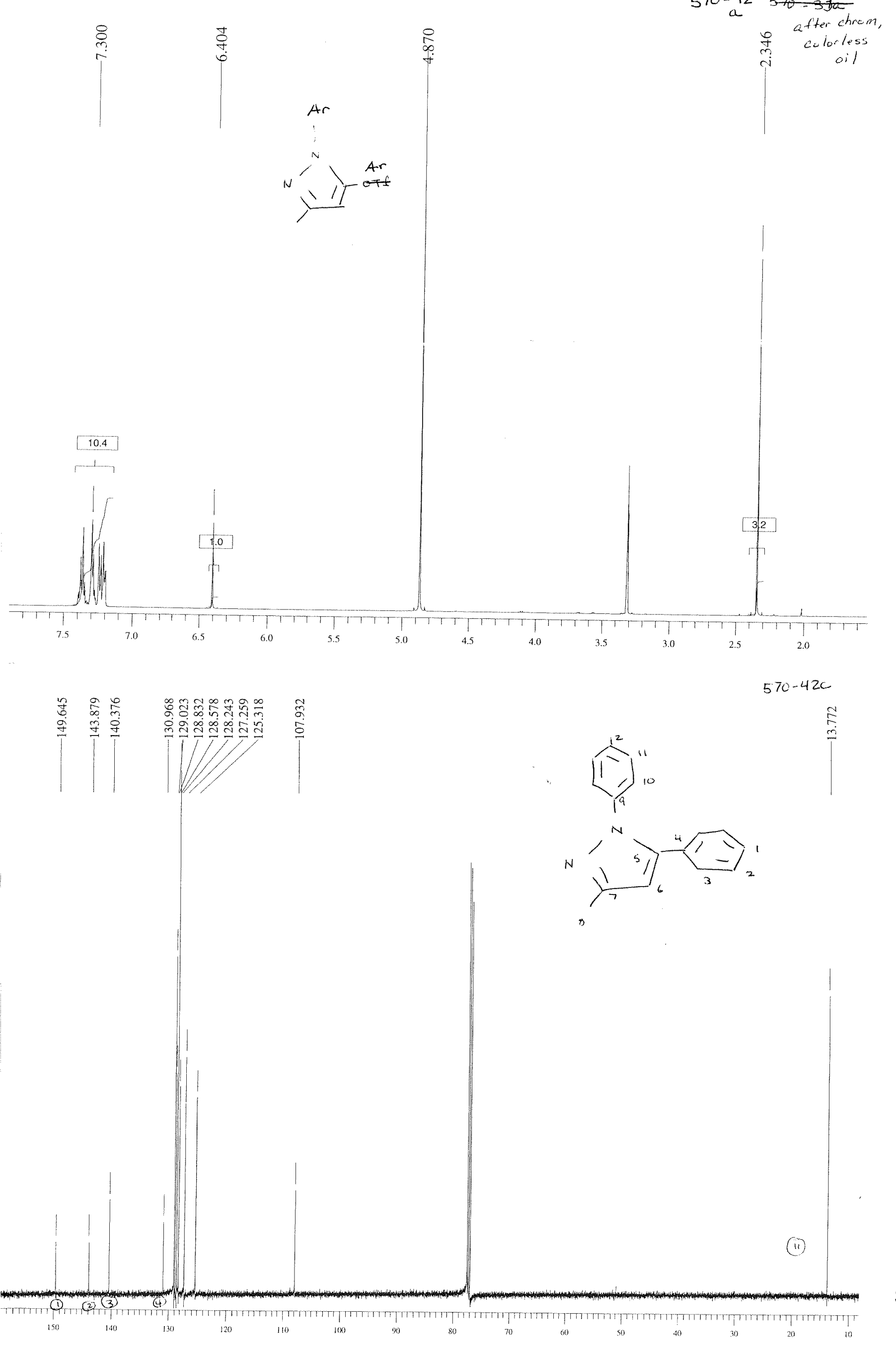

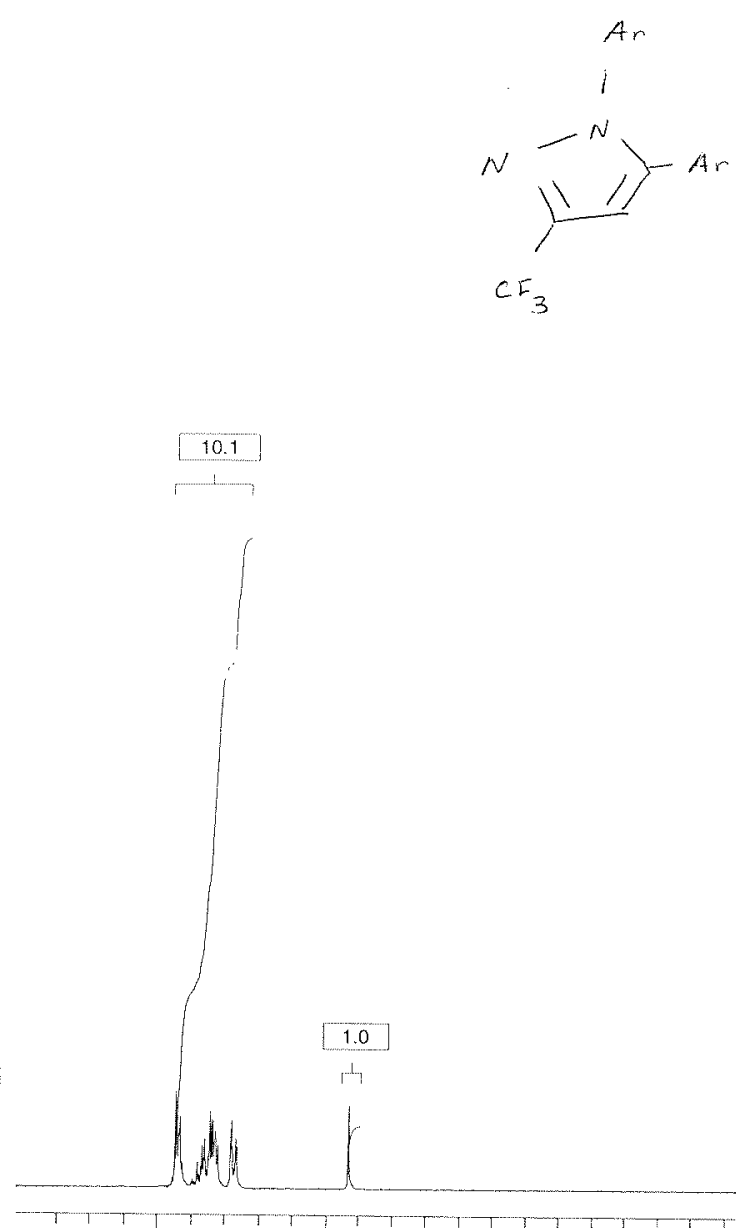

7.5

7.0

65

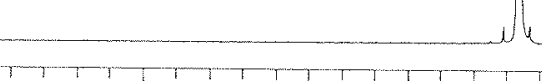

$\sqrt{4}$

5.5

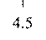

4.

3.0

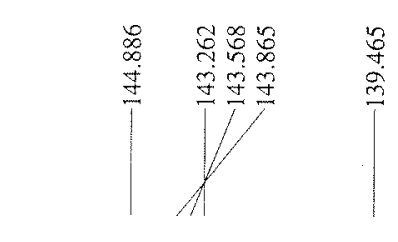

$570-40-2 c$

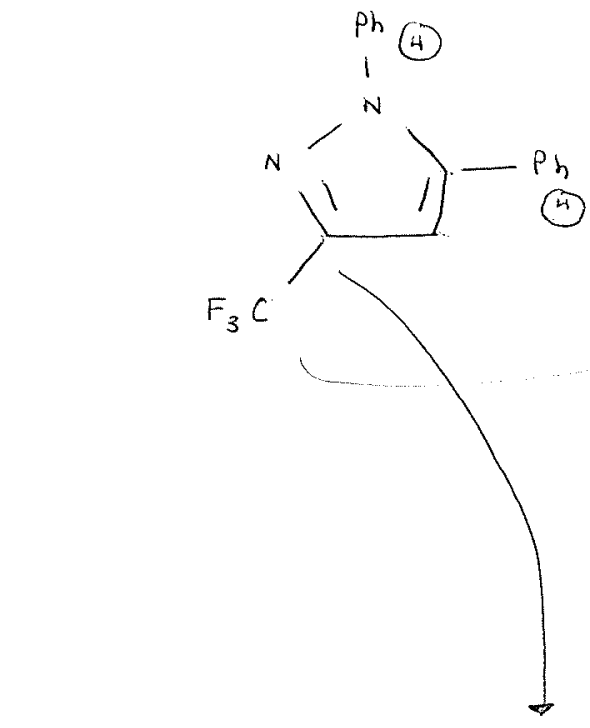

(4)
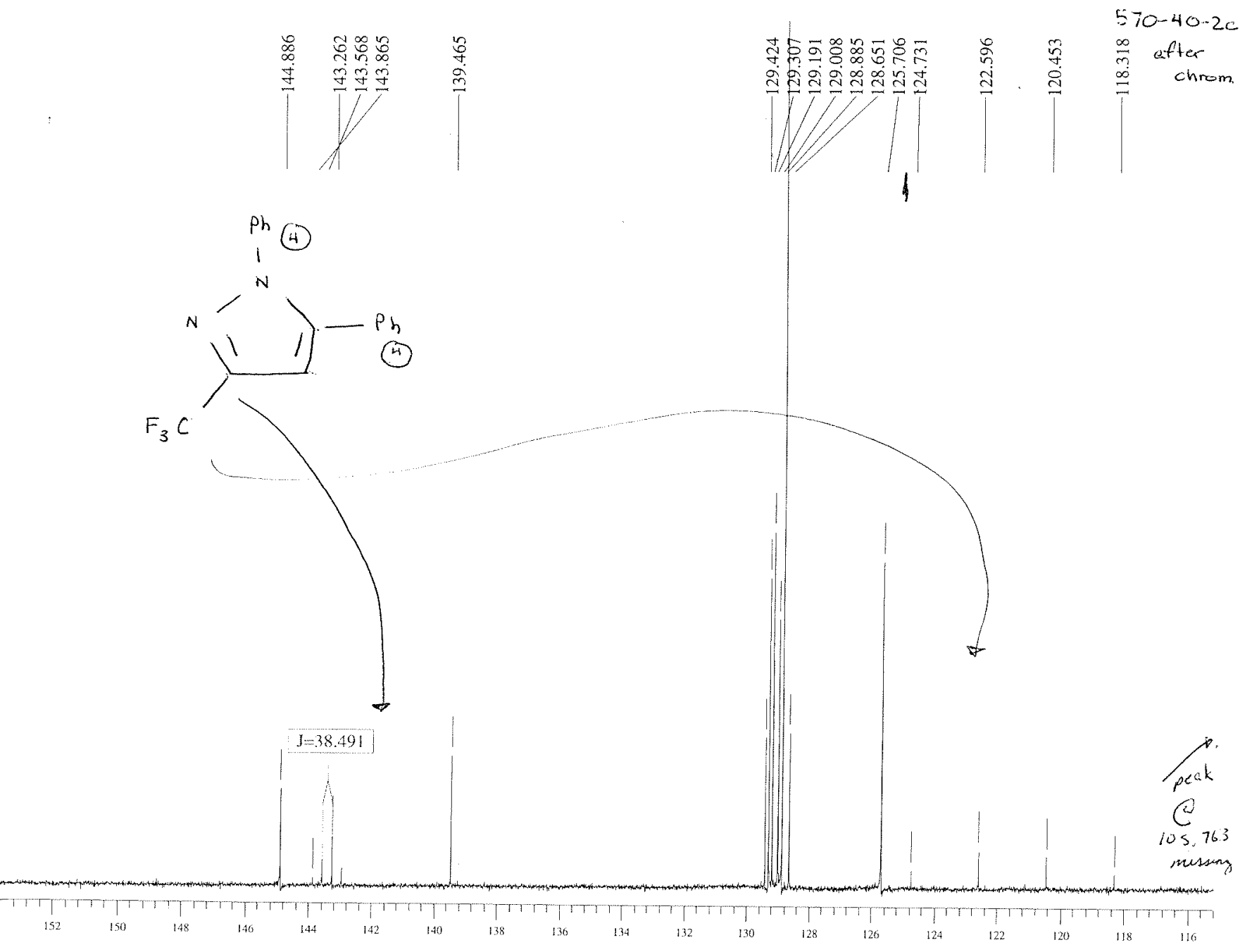


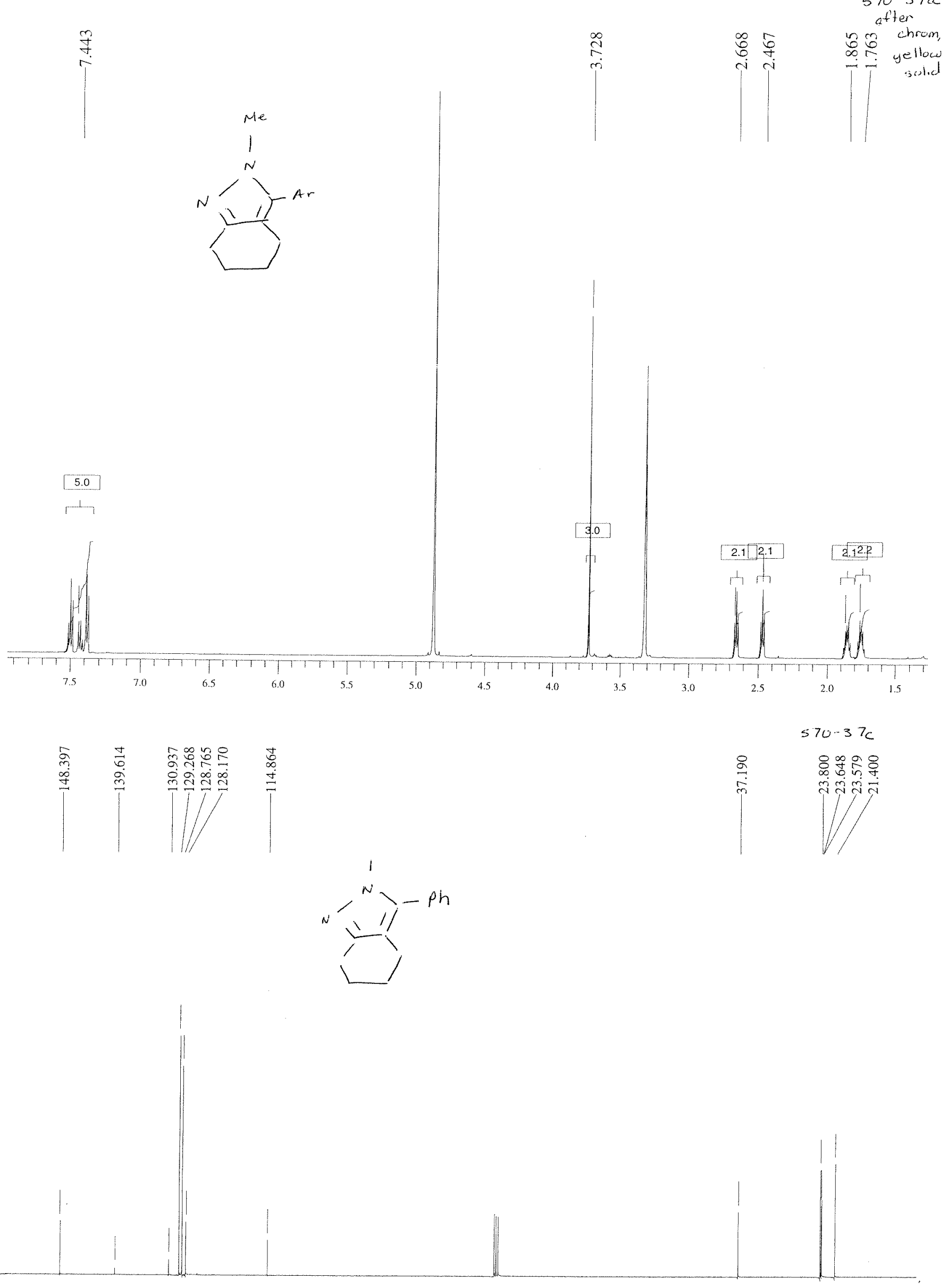




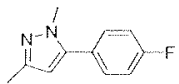

$2.0[2.0$
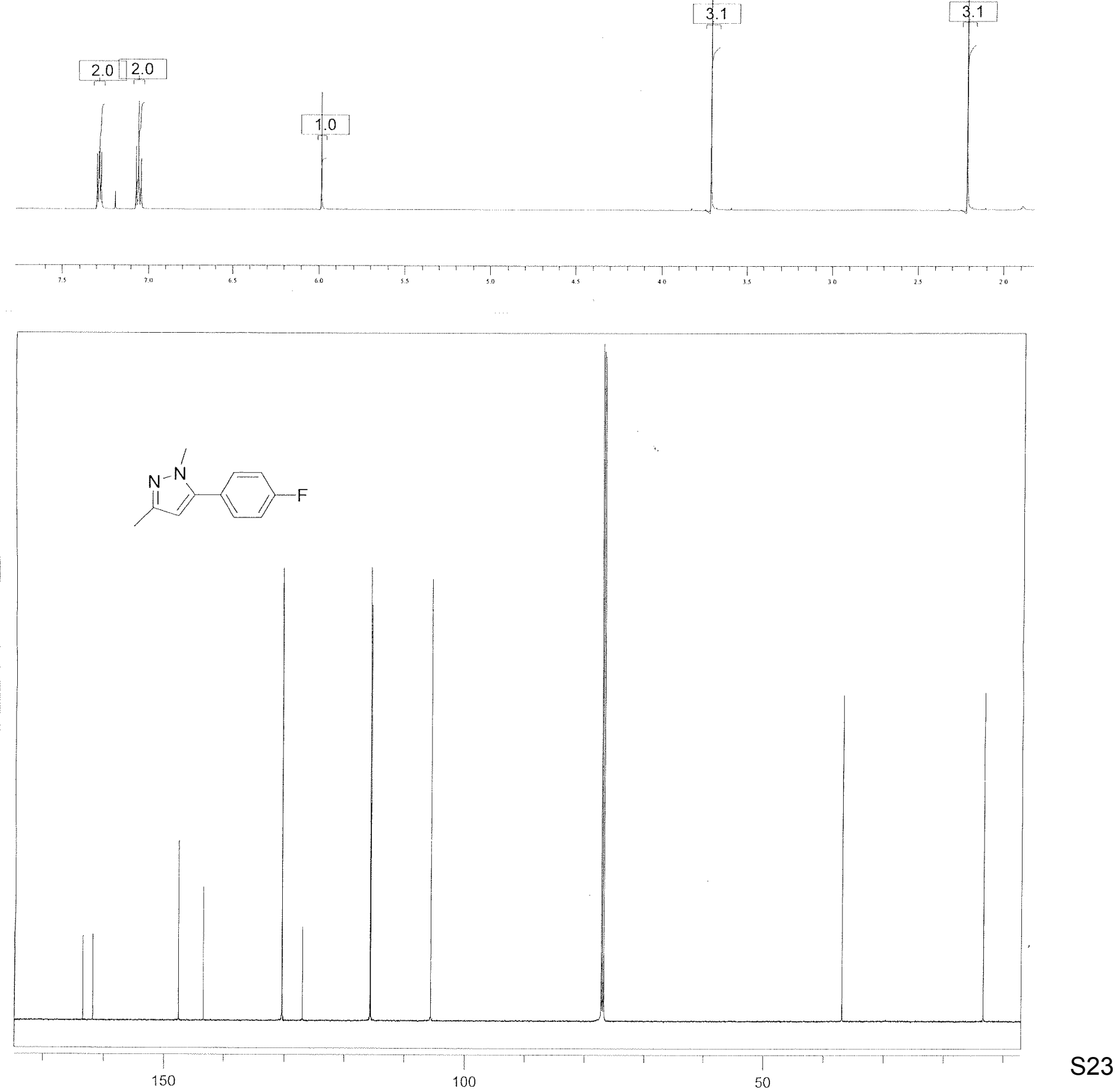

ppm (11)

100 

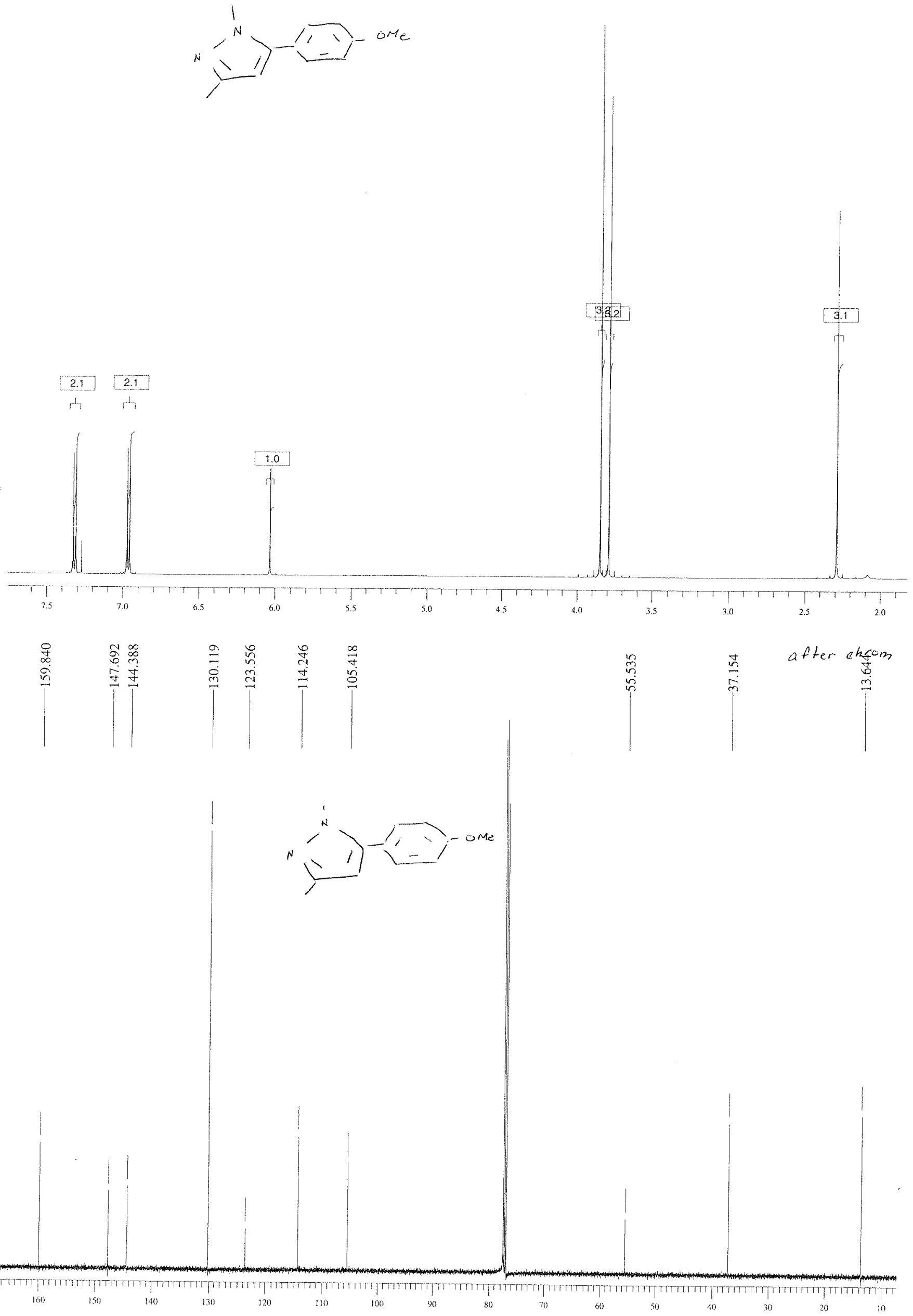
